\title{
TITCHMARSH DIVISOR PROBLEM FOR ABELIAN VARIETIES OF TYPES I, II, III, AND IV
}

\section{CRISTIAN VIRDOL}

Abstract. We study the Titchmarsh divisor problem in the context of abelian varieties. For abelian varieties of types I, II, III, and IV, under GRH, we obtain asymptotic formulas.

\section{INTRODUCTION}

Let $A$ be an abelian variety defined over a number field $F$, of conductor $\mathcal{N}$ and of dimension $r$, where $r \geq 1$ is an integer. Let $\Sigma_{F}$ be the set of finite places of $F$, and let $\mathcal{P}_{A}$ be the set of primes $\wp \in \Sigma_{F}$ of good reduction for $A$ (i.e. $\left(N_{F / \mathbb{Q}} \wp, N_{F / \mathbb{Q}} \mathcal{N}\right)=1$ ). For each positive integer $m$, let $A[m]$ be the set of $m$-torsion points of $A$.

Let $a$ be a positive integer. For $\wp \in \mathcal{P}_{A}$ we define

$$
\tau_{A, a, F}(\wp)=\left\{m \in \mathbb{N} \mid\left(m, N_{F / \mathbb{Q}} \wp\right)=1, \sigma_{\wp} \text { acts on } A[m] \simeq(\mathbb{Z} / m \mathbb{Z})^{2 r}\right.
$$

through the scalar matrix $\left.a \cdot I_{2 r}\right\}$,

where $\sigma_{\wp}$ denotes a Frobenius element at $\wp$ in $\operatorname{Gal}(F(A[m]) / F)$.

Since $F\left(\zeta_{m}\right) \subset F(A[m])$, using the Weil pairing on the abelian variety $A$ we get that the action of $\sigma_{\wp}$ on $F\left(\zeta_{m}\right)$ is given by $\zeta_{m} \mapsto \zeta_{m}^{a^{2}}$. Hence if $m \in \tau_{A, a, F}(\wp)$, then $N_{F / \mathbb{Q}} \wp \equiv a^{2}(\bmod m)$, and thus $\left|\tau_{A, a, F}(\wp)\right|<\infty$ for all $\wp \in \mathcal{P}_{A}$ such that $N_{F / \mathbb{Q} \wp}>a^{2}$ (in order to simplify our notation, and also because we are interested in asymptotic estimates, we assume these kinds of facts from now on).

For $x \in \mathbb{R}$ we define

$$
f_{A, a, F}(x):=\sum_{\substack{\wp \in \mathcal{P}_{A} \\ N_{F / \mathbb{Q} \wp \leq x}}}\left|\tau_{A, a, F}(\wp)\right| .
$$

In this paper we prove the following results (which are a geometric analog of the Titchmarsh division problem in the context of abelian varieties (see AG] for details)):

Theorem 1.1. Let $A$ be an abelian variety over a number field $F$ of dimension $r \geq 1$. Let a be a positive integer. Assume that the Generalized Riemann Hypothesis (GRH) holds for the Dedekind zeta functions of the division fields for A. Then we have

$$
f_{A, a, F}(x)=c_{A, F} l i x+O\left(x^{\frac{4 r^{2}-2 r+3}{4 r^{2}-2 r+4}}(\log x)^{\frac{2 r^{2}-r+1}{2 r^{2}-r+2}}\right),
$$

Received by the editors July 26, 2013 and, in revised form, July 28, 2013, July 30, 2013, August 2, 2013, February 12, 2014, March 16, 2014, February 26, 2015, March 1, 2015, and March 4, 2015.

2010 Mathematics Subject Classification. Primary 11G10, 11G15.

Key words and phrases. Abelian varieties, Titchmarsh divisor problem. 
where

$$
c_{A, F}=\sum_{m=1}^{\infty} \frac{1}{[F(A[m]): F]} .
$$

Theorem 1.2. Let $A$ be an abelian variety over a number field $F$ of dimension $r \geq$ 1 , such that $\operatorname{End}_{F} A \otimes \mathbb{Q}=E_{\bar{F}} A \otimes \mathbb{Q}$. Assume that i) $r=$ he and $\operatorname{End}_{\bar{F}} A \otimes \mathbb{Q}=E$ is a totally real number field, such that $[E: \mathbb{Q}]=e$, or that ii) $r=2$ he and $\operatorname{End}_{\bar{F}} A \otimes \mathbb{Q}=M$ is a totally indefinite quaternion division algebra over a totally real number field $E$, such that $[E: \mathbb{Q}]=e$, or that iii) $r=2$ he and $E_{\bar{F}} A \otimes \mathbb{Q}=M$ is a totally definite quaternion division algebra over a totally real number field $E$, such that $[E: \mathbb{Q}]=e$, where $h$ is an integer. Let a be a positive integer. Assume that GRH holds for the Dedekind zeta functions of the division fields for A. Then we have

$$
f_{A, a, F}(x)=c_{A, F} l i x+O\left(x^{\frac{4 h^{2}-2 h+3}{4 h^{2}-2 h+4}}(\log x)^{\frac{2 h^{2}-h+1}{2 h^{2}-h+2}}\right),
$$

where

$$
c_{A, F}=\sum_{m=1}^{\infty} \frac{1}{[F(A[m]): F]} .
$$

Theorem 1.3. Let $A$ be an abelian variety over a number field $F$ of dimension $r \geq$ 1 , such that $\operatorname{End}_{F} A \otimes \mathbb{Q}=\operatorname{End}_{\bar{F}} A \otimes \mathbb{Q}$. Assume that $r=$ hed and $\operatorname{End}_{\bar{F}} A \otimes \mathbb{Q}=M$ is a division algebra over a $C M$ number field $K$, such that $[K: \mathbb{Q}]=2 e$, and $[M: K]=d^{2}$, where $d, e$, and $h$ are integers. Let a be a positive integer. Assume that GRH holds for the Dedekind zeta functions of the division fields for A. Then we have

$$
f_{A, a, F}(x)=c_{A, F} l i x+O\left(x^{\frac{4 h^{2}-2 h+3}{4 h^{2}-2 h+4}}(\log x)^{\frac{2 h^{2}-h+1}{2 h^{2}-h+2}}\right),
$$

where

$$
c_{A, F}=\sum_{m=1}^{\infty} \frac{1}{[F(A[m]): F]} .
$$

Moreover when $e=1$ we have

$$
f_{A, a, F}(x)=c_{A, F} l i x+O\left(x^{\frac{2 h^{2}+2 h-1}{2 h^{2}+2 h}}(\log x)^{\frac{h^{2}+h-1}{h^{2}+h}}\right) .
$$

Theorem 1.4. Let $A$ be an abelian variety over a number field $F$ of dimension $r \geq 1$. Assume that i) $r=$ he and $\operatorname{End}_{\bar{F}} A \otimes \mathbb{Q}=E$ is a totally real number field, such that $[E: \mathbb{Q}]=e$ and $h$ is an odd integer, or that ii) $r=2$ he and $\operatorname{End}_{\bar{F}} A \otimes \mathbb{Q}=M$ is a totally indefinite quaternion division algebra over a totally real number field $E$, such that $[E: \mathbb{Q}]=e$ and $h$ is an odd integer. Let a be a positive integer. Assume that GRH, Artin's Holomorphy Conjecture (AHC), and Pair Correlation Conjecture (PCC) hold for the Artin L-functions attached to the irreducible characters of $\operatorname{Gal}(F(A[m]) / F)$ for any integer $m$. Then, for every $\epsilon>0$, we have

$$
f_{A, a, F}(x)=c_{A, F} \text { li } x+O\left(x^{\frac{2 r^{2}-r+2}{2}-\left(2 r^{2}-r+1\right) \frac{4 r^{2}-2 r+2}{8 r^{2}-4 r+8-2 e h^{2}+e h-e+\epsilon}}\right),
$$

where

$$
c_{A, F}=\sum_{m=1}^{\infty} \frac{1}{[F(A[m]): F]} .
$$


Theorem 1.5. Let $A$ be an abelian variety over a number field $F$ of dimension $r \geq 1$. Assume that $r=2$ he and $\operatorname{End}_{\bar{F}} A \otimes \mathbb{Q}=M$ is a totally definite quaternion division algebra over a totally real number field $E$, such that $[E: \mathbb{Q}]=e$, where $h$ is an odd integer. Let a be a positive integer. Assume that GRH, AHC and PCC hold for the Artin L-functions attached to the irreducible characters of $\operatorname{Gal}(F(A[m]) / F)$ for any integer $m$. Then, for every $\epsilon>0$, we have

$$
f_{A, a, F}(x)=c_{A, F} l i x+O\left(x^{\left.\frac{2 r^{2}-r+2}{2}-\left(2 r^{2}-r+1\right) \frac{4 r^{2}-2 r+2}{8 r^{2}-4 r+8-e h^{2}+e h+\epsilon}\right),}\right.
$$

where

$$
c_{A, F}=\sum_{m=1}^{\infty} \frac{1}{[F(A[m]): F]} .
$$

We remark that in Theorems 1.4 and 1.5 one could impose, as we did in Theorems 1.2 and 1.3 above, the condition $\operatorname{End}_{F} A \otimes \mathbb{Q}=\operatorname{End}_{\bar{F}} A \otimes \mathbb{Q}$ and in this case one obtains better asymptotic formulas (but the computations are not so nice).

All these results are generalizations of AG, where the authors were able to prove only the very particular case when the abelian variety $A$ is defined over $\mathbb{Q}$ and contains an abelian subvariety $E$ of dimension one also defined over $\mathbb{Q}$ (actually one can formulate many generalizations of [AG]; i.e. one can consider an abelian variety $A$ defined over a number field $F$, of arbitrary dimension, which contains an abelian subvariety $E$ defined over $F$ of arbitrary dimension and of arbitrary type, etc.). We remark that the exact same methods used in this paper could be extended to Drinfeld modules of arbitrary ranks.

\section{General abelian varieties}

For $F$ a number field, we denote $G_{F}:=\operatorname{Gal}(\bar{F} / F)$. Let $A$ be an abelian variety over $F$ of dimension $r \geq 1$ and of conductor $\mathcal{N}$. Let $\Sigma_{F}$ be the set of finite places of $F$, and for $\wp$ a prime of $F$, let $\mathbb{F}_{\wp}$ be the residue field at $\wp$. Let $\mathcal{P}_{A}$ be the set of primes $\wp \in \Sigma_{F}$ of good reduction for $A$ (i.e. $\left(N_{F / \mathbb{Q}} \wp, N_{F / \mathbb{Q}} \mathcal{N}\right)=1$ ). For $\wp \in \mathcal{P}_{A}$, we denote by $\bar{A}$ the reduction of $A$ at $\wp$. For $m \geq 1$ an integer, let $A[m]$ be the $m$-division points of $A$ in $\bar{F}$. Then

$$
A[m] \simeq(\mathbb{Z} / m \mathbb{Z})^{2 r} .
$$

If $F(A[m])$ is the field obtained by adjoining to $F$ the elements of $A[m]$, then we have a natural injection

$$
\Phi_{m}: \operatorname{Gal}(F(A[m]) / F) \hookrightarrow \operatorname{Aut}(A[m]) \simeq \mathrm{GL}_{2 r}(\mathbb{Z} / m \mathbb{Z})
$$

We denote $G_{m}:=\operatorname{Im} \Phi_{m}(\operatorname{Gal}(F(A[m]) / F))$. Define

$$
n(m):=\left|G_{m}\right|=[F(A[m]): F] .
$$

For a rational prime $l$, let

$$
T_{l}(A)=\lim _{\longleftarrow} A\left[l^{n}\right]
$$

and $V_{l}(A)=T_{l}(A) \otimes \mathbb{Q}$. The Galois group $G_{F}$ acts on

$$
T_{l}(A) \simeq \mathbb{Z}_{l}^{2 r},
$$

where $\mathbb{Z}_{l}$ is the $l$-adic completion of $\mathbb{Z}$ at $l$, and also on $V_{l}(A) \simeq \mathbb{Q}_{l}^{2 r}$, and we obtain a representation

$$
\rho_{A, l}: G_{F} \rightarrow \operatorname{Aut}\left(T_{l}(A)\right) \simeq \mathrm{GL}_{2 r}\left(\mathbb{Z}_{l}\right) \subset \operatorname{Aut}\left(V_{l}(A)\right) \simeq \mathrm{GL}_{2 r}\left(\mathbb{Q}_{l}\right),
$$


which is unramified outside $l N_{F / \mathbb{Q}} \mathcal{N}$. If $\wp \in \mathcal{P}_{A}$, let $\sigma_{\wp}$ be the Artin symbol of $\wp$ in $G_{F}$, and let $l$ be a rational prime satisfying $\left(l, N_{F / \mathbb{Q}} \wp\right)=1$. We denote by $P_{A, \wp}(X)=X^{2 r}+b_{1, A}(\wp) X^{2 r-1}+\ldots+b_{2 r-1, A}(\wp) X+N_{F / \mathbb{Q} \wp^{r}} \in \mathbb{Z}[X]$ the characteristic polynomial of $\sigma_{\wp}$ on $T_{l}(A)$. Then $P_{A, \wp}(X)$ is independent of $l$. One can identify $T_{l}(A)$ with $T_{l}(\bar{A})$, where $\bar{A}$ is the reduction of $A$ at $\wp$, and the action of $\sigma_{\wp}$ on $T_{l}(A)$ is the same as the action of the Frobenius $\pi_{\wp}$ of $\bar{A}$ on $T_{l}(\bar{A})$. For $a$ an integer define $P_{A, a, \wp}(X)=X^{2 r}+b_{1, A, a}(\wp) X^{2 r-1}+\ldots+b_{2 r-1, A, a}(\wp) X+b_{2 r, A, a}(\wp) \in$ $\mathbb{Z}[X]$ by $P_{A, a, \wp}(X):=P_{A, \wp}(X+a)$.

We know (see for example [SI]):

Lemma 2.1. Let $A$ be an abelian variety defined over a number field $F$, of dimension $r$, of conductor $\mathcal{N}$, and let $a$ and $m$ be positive integers. Then

1. The extension $F(A[m]) / F$ is ramified only at places dividing $m \mathcal{N}$,

2. $F\left(\zeta_{m}\right) \subseteq F(A[m])$. Hence if $\sigma_{\wp}$, for $\wp \in \mathcal{P}_{A}$ such that $\left(N_{F / \mathbb{Q} \wp, m)}=1\right.$, acts on $A[m] \simeq(\mathbb{Z} / m \mathbb{Z})^{2 r}$ through the scalar matrix $a \cdot I_{2 r}$, from the Weil pairing we get that $m \mid N_{F / \mathbb{Q} \wp}-a^{2}$.

We know (see (3.1) of $[\mathrm{AG}]$ ):

Lemma 2.2. Let $A$ be an abelian variety defined over a number field $F$. Let $\epsilon>0$. Then, with the same notation as above, we have

$$
\left|G_{m}\right| \gg m^{2-\epsilon} \text {. }
$$

Lemma 2.3. Let $A$ be an abelian variety over a number field $F$, of conductor $\mathcal{N}$. Let $\wp \in \mathcal{P}_{A}$, and let $p$ be the rational prime below $\wp$. Let $m$ be an integer relatively prime to $p$, and let a be a positive integer. If $\sigma_{\wp}$ acts on $A[m] \simeq(\mathbb{Z} / m \mathbb{Z})^{2 r}$ through the scalar matrix $a \cdot I_{2 r}$, then

$$
m^{k} \mid b_{k, A, a}(\wp)
$$

for any $k=1, \ldots, 2 r$.

Proof. Let $l \mid m$ be a rational prime, and let $m(l)$ be the largest natural number such that $l^{m(l)} \mid m$. Let

$$
\pi_{\wp}: \bar{A}\left(\overline{\mathbb{F}}_{\wp}\right) \rightarrow \bar{A}\left(\overline{\mathbb{F}}_{\wp}\right)
$$

be the Frobenius endomorphism at $\wp$. From the hypothesis we know that $\bar{A}\left(\overline{\mathbb{F}}_{\wp}\right)\left[l^{m(l)}\right] \subset \operatorname{Ker}\left(\pi_{\wp}-a\right)$, and we get that $\rho_{A, l}\left(\sigma_{\wp}\right)=a I_{2 r}+l^{m(l)} B_{l}$, where $B_{l} \in M_{2 r}\left(\mathbb{Z}_{l}\right)$. Thus $X^{2 r}+b_{1, A, a}(\wp) X^{2 r-1}+\ldots+b_{2 r-1, A, a}(\wp) X+b_{2 r, A, a}(\wp)=$ $P_{A, a, \wp}(X)=P_{A, \wp}(X+a)=\operatorname{det}\left((X+a) I_{2 r}-\rho_{A, l}\left(\sigma_{\wp}\right)\right)=\operatorname{det}\left(X I_{2 r}-l^{m(l)} B_{l}\right)$, and we obtain that $l^{m(l) k} \mid b_{k, A, a}(\wp)$, for any $k=1, \ldots, 2 r$, and we conclude the proof of Lemma 2.3.

Lemma 2.4. Let a be a positive integer. Then we have

$$
\left|b_{k, A, a}(\wp)\right| \leq(2+2 a)^{2 r} N_{F / \mathbb{Q}} \wp^{\frac{k}{2}},
$$

for any $k=1, \ldots, 2 r$.

Proof. We know (Riemann Hypothesis) that

$$
P_{A, \wp}(X)=\left(X-x_{1, \wp}\right) \ldots\left(X-x_{2 r, \wp}\right) \text {, }
$$

where $\left|x_{i, \wp}\right|=N_{F / \mathbb{Q} \wp} \wp^{\frac{1}{2}}$. Hence $X^{2 r}+b_{1, A, a}(\wp) X^{2 r-1}+\ldots+b_{2 r-1, A, a}(\wp) X+$ $b_{2 r, A, a}(\wp)=P_{A, \wp, a}(X)=P_{A, \wp}(X+a)=\left(X-\left(x_{1, \wp}-a\right)\right) \ldots\left(X-\left(x_{2 r, \wp}-a\right)\right)$, from 


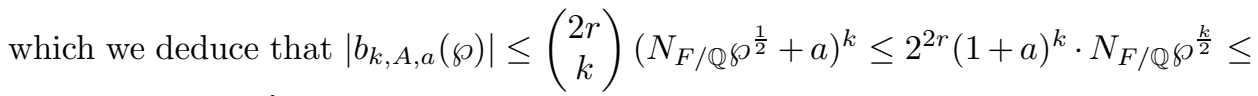
$(2+2 a)^{2 r} N_{F / \mathbb{Q}} \wp^{\frac{k}{2}}$, for any $k=1, \ldots, 2 r$.

Lemma 2.5. We have

$$
b_{2 r, A, a}(\wp)=N_{F / \mathbb{Q}} \wp^{r}+c_{1} b_{1, A, a}(\wp)+c_{2} b_{2, A, a}(\wp)+\ldots+c_{2 r-1} b_{2 r-1, A, a}(\wp)+c_{2 r},
$$

where $c_{1}, \ldots, c_{2 r}$ are integers which depend only on $r$ and $a$.

Proof. From $P_{A, \wp, a}(X):=P_{A, \wp}(X+a)$, we get

$$
\begin{aligned}
& b_{1, A, a}(\wp)=b_{1, A}(\wp)+a\left(\begin{array}{c}
2 r \\
1
\end{array}\right), \\
& b_{2, A, a}(\wp)=b_{2, A}(\wp)+b_{1, A}(\wp) a\left(\begin{array}{c}
2 r-1 \\
1
\end{array}\right)+a^{2}\left(\begin{array}{c}
2 r \\
2
\end{array}\right) \text {, } \\
& b_{2 r, A, a}(\wp)=N_{F / \mathbb{Q} \wp^{r}}+b_{2 r-1, A}(\wp) a\left(\begin{array}{l}
1 \\
1
\end{array}\right)+\ldots+a^{2 r}\left(\begin{array}{l}
2 r \\
2 r
\end{array}\right),
\end{aligned}
$$

and by writing $b_{1, A}(\wp)$ in terms of $b_{1, A, a}(\wp)$, then $b_{2, A}(\wp)$ in terms of $b_{2, A, a}(\wp)$ and $b_{1, A, a}(\wp), \ldots$, and $N_{F / \mathbb{Q}} \wp^{r}$ in terms of $b_{1, A, a}(\wp), \ldots$ and $b_{2 r, A, a}(\wp)$, we are done with the proof of Lemma 2.5 .

\section{Abelian VARIETIES OF tYPes I, II AND III}

Let $A$ be an abelian variety of dimension $r$, defined over a number field $F$, such that $\operatorname{End}_{F} A \otimes \mathbb{Q}=\operatorname{End} A_{\bar{F}} \otimes \mathbb{Q}$. Assume that i) $r=h e$ and $\operatorname{End}_{\bar{F}} A \otimes \mathbb{Q}=E$ is a totally real number, such that $[E: \mathbb{Q}]=e$, or that ii) $r=2 h e$ and $\operatorname{End}_{\bar{F}} A \otimes \mathbb{Q}=M$ is a totally indefinite quaternion division algebra over a totally real number field $E$, such that $[E: \mathbb{Q}]=e$, or that iii) $r=2 h e$ and $\operatorname{End}_{\bar{F}} A \otimes \mathbb{Q}=M$ is a totally definite quaternion division algebra over a totally real number field $E$, such that $[E: \mathbb{Q}]=e$, where $h$ is an integer. Let $O_{E}$ be the ring of integers of $E$, and let $\left\{\sigma_{1}, \ldots, \sigma_{e}\right\}$ be the set of all embeddings of $E$ into $\mathbb{C}$.

Let $l$ be a rational prime. Since the actions of $M:=\operatorname{End}_{F} A \otimes \mathbb{Q}$ and $G_{F}$ on $V_{l}(A)$ commute we obtain a $2 h$-dimensional representation

$$
\rho_{l}: G_{F} \rightarrow \operatorname{Aut}_{M_{l}} V_{l}(A) \cong \mathrm{GL}_{2 h}\left(E_{l}\right),
$$

where $M_{l}:=\operatorname{End}_{F} A \otimes \mathbb{Q}_{l}$ and $E_{l}:=E \otimes \mathbb{Q}_{l}$. We denote by $Q_{A, \wp}(X)=X^{2 h}+$ $c_{1, A}(\wp) X^{2 h-1}+\ldots+c_{2 h-1, A}(\wp) X+N_{F / \mathbb{Q}} \wp^{h} \in O_{E}[X]$ the characteristic polynomial of $\sigma_{\wp}$ on $V_{l}(A)$. For $a \in \mathbb{Z}$ define $Q_{A, \wp, a}(X)=X^{2 h}+c_{1, A, a}(\wp) X^{2 h-1}+\ldots+$ $c_{2 h-1, A, a}(\wp) X+c_{2 h, A, a}(\wp) \in O_{E}[X]$ by $Q_{A, \wp, a}(X):=Q_{A, \wp}(X+a)$.

Lemma 3.1. Let $A$ be an abelian variety over a number field $F$, as above. Let $\wp \in \mathcal{P}_{A}$, and let $m$ be a positive integer such that $\left(N_{F / \mathbb{Q} \wp, m)}=1\right.$. Let a be a positive integer. If $\sigma_{\wp}$ acts on $A[m] \simeq(\mathbb{Z} / m \mathbb{Z})^{2 r}$ through the scalar matrix $a \cdot I_{2 r}$, then

$$
m^{k} \mid c_{k, A, a}(\wp)
$$

for any $k=1, \ldots, 2 h$. 
Proof. Let $l \mid m$ be a rational prime, and let $m(l)$ be the largest natural number such that $l^{m(l)} \mid m$. Let

$$
\pi_{\wp}: \bar{A}\left(\overline{\mathbb{F}}_{\wp}\right) \rightarrow \bar{A}\left(\overline{\mathbb{F}}_{\wp}\right)
$$

be the Frobenius endomorphism at $\wp$. From the hypothesis we know that $\bar{A}\left(\overline{\mathbb{F}}_{\wp}\right)\left[l^{m(l)}\right] \subset \operatorname{Ker}\left(\pi_{\wp}-a\right)$, and we get that $\rho_{A, l}\left(\sigma_{\wp}\right)=a I_{2 h}+l^{m(l)} B_{l}$, where $B_{l} \in M_{2 h}\left(O_{E} \otimes \mathbb{Z}_{l}\right)$. Thus $X^{2 h}+c_{1, A, a}(\wp) X^{2 h-1}+\ldots+c_{2 h-1, A, a}(\wp) X+c_{2 h, A, a}(\wp)=$ $Q_{A, a, \wp}(X)=Q_{A, \wp}(X+a)=\operatorname{det}\left((X+a) I_{2 h}-\rho_{A, l}\left(\sigma_{\wp}\right)\right)=\operatorname{det}\left(X I_{2 h}-l^{m(l)} B_{l}\right)$, and we obtain that $l^{m(l) k} \mid c_{k, A, a}(\wp)$, for any $k=1, \ldots, 2 h$, concluding the proof of Lemma 3.1.

Lemma 3.2. Let a be a positive integer. Then we have

$$
\left|\sigma_{i}\left(c_{k, A, a}(\wp)\right)\right| \leq(2+2 a)^{2 r} N_{F / \mathbb{Q}} \wp^{\frac{k}{2}},
$$

for any $i=1, \ldots, e$ and for any $k=1, \ldots, 2 h$.

Proof. We assume that $\sigma_{i}$ is the trivial embedding of $E$ into $\mathbb{C}$ and prove Lemma 3.2 in this case. We know (Riemann Hypothesis) that

$$
Q_{A, \wp}(X)=\left(X-y_{1, \wp}\right) \ldots\left(X-y_{2 h, \wp}\right),
$$

where $\left|y_{i, \wp}\right|=N_{F / \mathbb{Q} \wp^{\frac{1}{2}}}$. Hence $X^{2 h}+c_{1, A, a}(\wp) X^{2 h-1}+\ldots+c_{2 h-1, A, a}(\wp) X+$ $c_{2 h, A, a}(\wp)=Q_{A, \wp, a}(X)=Q_{A, \wp}(X+a)=\left(X-\left(y_{1, \wp}-a\right)\right) \ldots\left(X-\left(y_{2 h, \wp}-a\right)\right)$, from which we deduce, as in the proof of Lemma 2.4 above, that $\left|c_{k, A, a}(\wp)\right| \leq$ $(2+2 a)^{2 r} N_{F / \mathbb{Q} \wp^{\frac{k}{2}}}$, for any $k=1, \ldots, 2 r$. When $\sigma_{i}$ is not the trivial embedding of $E$ into $\mathbb{C}$ the proof of Lemma 3.2 is similar.

Lemma 3.3. We have

$c_{2 h, A, a}(\wp)=N_{F / \mathbb{Q} \wp^{h}+d_{1, a}} c_{1, A, a}(\wp)+d_{2, a} c_{2, A, a}(\wp)+\ldots+d_{2 h-1, a} c_{2 h-1, A, a}(\wp)+d_{2 h, a}$, where $d_{1, a}, \ldots, d_{2 h, a}$ are integers which depend only on $h$ and $a$.

Proof. The proof is similar to the proof of Lemma 2.5.

Lemma 3.4. With the same notation as above, for any $m \in \mathbb{N}^{*}$ and any $x \in \mathbb{R}$, we have

$$
\begin{gathered}
\pi_{a}(x, F(A[m]) / F):=\mid\left\{\wp \in \mathcal{P}_{A} \mid N_{F / \mathbb{Q} \wp} \leq x,\left(m, N_{F / \mathbb{Q} \wp}\right)=1,\right. \\
\left.\sigma_{\wp} \text { acts on } A[m] \simeq(\mathbb{Z} / m \mathbb{Z})^{2 r} \text { through the scalar matrix } a \cdot I_{2 r}\right\} \mid \\
\ll \frac{x^{\frac{2 h^{2}-h+2}{2}}}{m^{2 h^{2}-h+2}}+1 .
\end{gathered}
$$

Proof. Let $\wp \in \mathcal{P}_{A}$ be such that $N_{F / \mathbb{Q}} \wp \leq x,\left(m, N_{F / \mathbb{Q}} \wp\right)=1$, and $\sigma_{\wp}$ acts on $A[m] \simeq(\mathbb{Z} / m \mathbb{Z})^{2 r}$ through the scalar matrix $a \cdot I_{2 r}$. Then from Lemma 3.3 we know that $c_{2 h, A, a}(\wp)=N_{F / \mathbb{Q} \wp^{h}}+d_{1, a} c_{1, A, a}(\wp)+d_{2, a} c_{2, A, a}(\wp)+\ldots+d_{2 h-1, a} c_{2 h-1, A, a}(\wp)+$ $d_{2 h, a}$, and hence

$$
\begin{aligned}
\sum_{i=1}^{e} \sigma_{i}\left(c_{2 h, A, a}(\wp)\right)= & e N_{F / \mathbb{Q}} \wp^{h}+d_{1, a} \sum_{i=1}^{e} \sigma_{i}\left(c_{1, A, a}(\wp)\right) \\
& +\ldots+d_{2 h-1, a} \sum_{i=1}^{e} \sigma_{i}\left(c_{2 h-1, A, a}(\wp)\right)+e d_{2 h, a} .
\end{aligned}
$$


Also, from Lemma 3.1 we know that $m^{k} \mid \sum_{i=1}^{e} \sigma_{i}\left(c_{k, A, a}(\wp)\right)$, for any $k=1, \ldots, 2 h$, and from Lemma 3.2 we know that $\left|\sum_{i=1}^{e} \sigma_{i}\left(c_{k, A, a}(\wp)\right)\right| \leq e(2+2 a)^{2 r} N_{F / \mathbb{Q}} \wp^{\frac{k}{2}} \ll$ $x^{\frac{k}{2}}$, for any $k=1, \ldots, 2 h$. Thus using the fact that $\sum_{i=1}^{e} \sigma_{i}\left(c_{k, A, a}(\wp)\right)$, for $k=1, \ldots, 2 h$, are integers, we get that the number of $\sum_{i=1}^{e} \sigma_{i}\left(c_{k, A, a}(\wp)\right)$ is $\ll\left(\frac{x^{\frac{k}{2}}}{m^{k}}+1\right)$, for $k=1, \ldots, 2 h$. Hence, since $N_{F / \mathbb{Q}} \wp \leq x$, and because $m^{2 h} \mid e N_{F / \mathbb{Q} \wp^{h}}+d_{1, a} \sum_{i=1}^{e} \sigma_{i}\left(c_{1, A, a}(\wp)\right)+\ldots+d_{2 h-1, a} \sum_{i=1}^{e} \sigma_{i}\left(c_{2 h-1, A, a}(\wp)\right)+e d_{2 h, a}$, we get that (we remark also that there are at most $[F: \mathbb{Q}]$ of primes $\wp$ for which the numbers $N_{F / \mathbb{Q} \wp}$ are equal):

$$
\begin{gathered}
\pi_{a}(x, F(A[m]) / F) \ll\left(\frac{x}{m^{2}}+1\right) \prod_{k=1}^{2 h-1}\left(\frac{x^{\frac{k}{2}}}{m^{k}}+1\right) \\
\ll\left(\frac{x}{m^{2}}+1\right)\left(\frac{x^{\frac{2 h^{2}-h}{2}}}{m^{2 h^{2}-h}}+1\right) \\
\ll \frac{x^{\frac{2 h^{2}-h+2}{2}}}{m^{2 h^{2}-h+2}}+1 .
\end{gathered}
$$

Lemma 3.5. Let $A$ be an abelian variety over a number field $F$ of dimension $r \geq 1$. Assume that i) $r=$ he and $E n d_{\bar{F}} A \otimes \mathbb{Q}=E$ is a totally real number field, such that $[E: \mathbb{Q}]=e$ and $h$ is an odd integer, or that ii) $r=2$ he and $\operatorname{End}_{\bar{F}} A \otimes \mathbb{Q}=M$ is a totally indefinite quaternion division algebra over a totally real number field $E$, such that $[E: \mathbb{Q}]=e$ and $h$ is an odd integer. Then for every $\epsilon>0$ we have

$$
m^{e\left(2 h^{2}+h\right)+1-\epsilon} \ll\left|G_{m}\right| \leq m^{e\left(2 h^{2}+h\right)+1} .
$$

Proof. By eventually replacing $F$ by a finite extension, one can assume that $\operatorname{End}_{F} A$ $\otimes \mathbb{Q}=\operatorname{End}_{\bar{F}} A \otimes \mathbb{Q}$, and we do assume this from now on. Then, as above we have an injective map (see BGK1] for details)

$$
\bar{\rho}_{m}: G_{m} \hookrightarrow \operatorname{GSp}_{2 h}\left(O_{E} / m O_{E}\right) .
$$

Since $\left|\mathrm{Sp}_{2 h}\left(O_{E} / m O_{E}\right)\right| \leq m^{e\left(2 h^{2}+h\right)}$ (we remark also that $\left|\operatorname{Sp}_{2 h}\left(O_{E} / m O_{E}\right)\right|$ is a monic "polynomial" in $m$ of degree $e\left(2 h^{2}+h\right)$; for us even $\varphi(m)=$ $m \prod_{l \mid m}\left(1-\frac{1}{l}\right)$ is a monic polynomial of degree one, so all we are interested in in the proofs below is that there exist positive constants $c$ and $d$ such that $m^{e\left(2 h^{2}+h\right)} c^{\nu(m)}$ $\ll\left|\operatorname{Sp}_{2 h}\left(O_{E} / m O_{E}\right)\right| \ll m^{e\left(2 h^{2}+h\right)} d^{\nu(m)}$, where $\nu(m)$ is the number of prime divisors of $m$ ), we get easily (see also the beginning of the proof of Theorem 7.42 of [BGK1]) that

$$
\left|G_{m}\right| \leq m^{e\left(2 h^{2}+h\right)+1},
$$

and we are done with the proof of the inequality on the right hand side of Lemma 3.5 .

Now we prove that there exists a positive constant $C$ such that

$$
\frac{m^{e\left(2 h^{2}+h\right)+1}}{C^{\nu(m)}}<\left|G_{m}\right|,
$$

for any positive integer $m$, where $\nu(m)$ is the number of distinct primes dividing $m$. From Theorem 1 of [SE1, after replacing $F$ by a finite extension, we obtain that the function

$$
l^{d} \mapsto\left[F\left(A\left[l^{d}\right]\right): F\right]
$$


is multiplicative in $l$, where $l$ runs over the rational primes ( $d$ stands for arbitrary powers of $l$ ). Hence it is sufficient to prove that there exists a positive constant $C$ such that for any rational prime $l$ and any non-negative integer $d$ we have

$$
\frac{l^{d\left(e\left(2 h^{2}+h\right)+1\right)}}{C}<\left|G_{l^{d}}\right| .
$$

But this fact is known, and it follows from the open image theorems of the $l$-adic representations associated with our abelian varieties, i.e. the abelian varieties that appear in BGK1] (more exactly see Theorem E and Theorem 7.38, and also the beginning of the proof of Theorem 7.42 of [BGK1]).

Now, because for any $\epsilon>0$ we have $C^{\nu(m)} \ll m^{\epsilon}$, from above we get that

$$
m^{e\left(2 h^{2}+h\right)+1-\epsilon} \ll\left|G_{m}\right|,
$$

and we are done with the proof of Lemma 3.5.

Corollary 3.6. Under the same assumptions as in Lemma 3.5 for every $\epsilon>0$ we have

$$
m^{-e\left(2 h^{2}-h+1\right)-\epsilon} \ll \frac{\left|\tilde{G}_{m}\right|}{\left|G_{m}\right|} \ll m^{-e\left(2 h^{2}-h+1\right)+\epsilon},
$$

where $\tilde{G}_{m}$ denotes the set of conjugacy classes of $G_{m}$.

Proof. The set of conjugacy classes in $\mathrm{Sp}_{2 h}\left(O_{E} / m O_{E}\right)$ is a "polynomial" in $m$ of degree $2 h e-e$. Then because of the open image theorems from BGK1 that we mentioned in the proof of Theorem 3.5, one can deduce easily Corollary 3.6.

Lemma 3.7. Let $A$ be an abelian variety over a number field $F$ of dimension $r \geq 1$. Assume that $r=2$ he and $\operatorname{End}_{\bar{F}} A \otimes \mathbb{Q}=M$ is a totally definite quaternion division algebra over a totally real number field $E$, such that $[E: \mathbb{Q}]=e$, where $h$ is an odd integer. Then for every $\epsilon>0$ we have

$$
m^{e h^{2}+1-\epsilon} \ll\left|G_{m}\right| \leq m^{e h^{2}+1} .
$$

Proof. By eventually replacing $F$ by a finite extension, one can assume that $\operatorname{End}_{F} A$ $\otimes \mathbb{Q}=\operatorname{End}_{\bar{F}} A \otimes \mathbb{Q}$, and we do assume this from now on.

As above we have an injective map (see [BGK2] for details)

$$
\bar{\rho}_{m}: G_{m} \hookrightarrow \mathrm{GO}_{2 h}\left(O_{E} / m O_{E}\right) .
$$

Since $\left|\mathrm{SO}_{2 h}\left(O_{E} / m O_{E}\right)\right| \leq m^{e h^{2}}$ (we remark also that $\left|\mathrm{SO}_{2 h}\left(O_{E} / m O_{E}\right)\right|$ is a monic "polynomial" in $m$ of degree $\left.e h^{2}\right)$, as in the proof of Lemma 3.5 above, we get that

$$
\left|G_{m}\right| \leq m^{e h^{2}+1}
$$

and we are done with the proof of the inequality on the right hand side of Lemma 3.7. The rest of Lemma 3.7 could be proved in the same way as Lemma 3.5 by using this time Theorems 7.2 and 5.23 of BGK2] instead of Theorems E and 7.38 of [BGK1].

Corollary 3.8. Under the same assumptions as in Lemma 3.7 for every $\epsilon>0$ we have

$$
m^{-e h^{2}+e h-\epsilon} \ll \frac{\left|\tilde{G}_{m}\right|}{\left|G_{m}\right|} \ll m^{-e h^{2}+e h+\epsilon},
$$

where $\tilde{G}_{m}$ denotes the set of conjugacy classes of $G_{m}$. 
Proof. Since the set of conjugacy classes in $\mathrm{SO}_{2 h}\left(O_{E} / m O_{E}\right)$ is a "polynomial" in $m$ of degree $h e$, and $\left|\mathrm{SO}_{2 h}\left(O_{E} / m O_{E}\right)\right|$ is a monic "polynomial" in $m$ of degree $e h^{2}$, one can prove Corollary 3.8 by the same argument as in the proof of Corollary 3.6 .

\section{Abelian varieties of type IV}

Let $A$ be an abelian variety of dimension $r$, defined over a number field $F$, such that $\operatorname{End}_{F} A \otimes \mathbb{Q}=\operatorname{End} A_{\bar{F}} \otimes \mathbb{Q}$. Assume that $\operatorname{End}_{\bar{F}}(A) \otimes \mathbb{Q}=M$, where $M$ is a division algebra over a CM number field $K$. Then $r=$ hed where $[M: K]=d^{2}$ and $[K: \mathbb{Q}]=2 e$, where $d, e$ and $h$ are integers (note that because we always have in the case of abelian varieties $[M: \mathbb{Q}] \mid 2 r$, we get that $d \mid h$ (see $[\mathrm{M}])$ ). Let $\sigma_{1}, \ldots, \sigma_{2 e}$ be the set of embeddings of $K$ into $\mathbb{C}$. Let $O_{K}$ be the ring of integers of $K$, and let $E$ be the maximal totally real subfield of $K$. Then obviously $[K: E]=2$.

Let $l$ be a rational prime. Since the actions of $M:=\operatorname{End}_{F} A \otimes \mathbb{Q}$ and $G_{F}$ on $V_{l}(A)$ commute we obtain a $2 h$-dimensional representation

$$
\rho_{l}: G_{F} \rightarrow \operatorname{Aut}_{M_{l}} V_{l}(A) \cong \mathrm{GL}_{2 h}\left(K_{l}\right),
$$

where $M_{l}:=\operatorname{End}_{F} A \otimes \mathbb{Q}_{l}$, and $K_{l}:=K \otimes \mathbb{Q}_{l}$. We denote by $Q_{A, \wp}(X)=X^{2 h}+$ $c_{1, A}(\wp) X^{2 h-1}+\ldots+c_{2 h-1, A}(\wp) X+N_{F / \mathbb{Q}} \wp^{h} \in O_{K}[X]$ the characteristic polynomial of $\sigma_{\wp}$ on $V_{l}(A)$. For $a \in \mathbb{Z}$ define $Q_{A, \wp, a}(X)=X^{2 h}+c_{1, A, a}(\wp) X^{2 h-1}+\ldots+$ $c_{2 h-1, A, a}(\wp) X+c_{2 h, A, a}(\wp) \in O_{K}[X]$ by $Q_{A, \wp, a}(X):=Q_{A, \wp}(X+a)$. Then one can prove similar results as in Lemmas 3.1, 3.2, and 3.3 for abelian varieties of type IV.

We say that $\wp \in \mathcal{P}_{A}$ is a prime of supersingular good reduction for $A$ if $Q_{A, \wp}(X)=$ $\left(x^{2}+N_{F / \mathbb{Q}} \wp\right)^{h}$; otherwise we say that $\wp \in \mathcal{P}_{A}$ is a prime of ordinary good reduction for $A$. If $\wp \in \mathcal{P}_{A}$ is a prime of ordinary good reduction for $A$, then

$$
\rho\left(\sigma_{\wp}\right)=\left(\begin{array}{cc}
C_{\wp} & 0 \\
0 & \bar{C}_{\wp}^{t}
\end{array}\right),
$$

where $C_{\wp} \in \mathrm{GL}_{h}\left(O_{K} \otimes \mathbb{Z}_{l}\right)$ and $\bar{C}_{\wp}^{t}$ is the complex conjugate transpose of $C_{\wp}$ (the complex conjugation is taken with respect to $K / E$; see [M] and [SI] for details, and for the theory of $l$-adic representations associated to abelian varieties of types I, II, III see BGK1] and BGK2]). We denote by $R_{A, \wp}(X)=X^{h}+d_{1, A}(\wp) X^{h-1}+\ldots+$ $d_{h-1, A}(\wp) X+d_{h, A}(\wp) \in O_{K}[X]$ the characteristic polynomial of $C_{\wp}$. For $a \in \mathbb{Z}$ define $R_{A, \wp, a}(X)=X^{h}+d_{1, A, a}(\wp) X^{h-1}+\ldots+d_{h-1, A, a}(\wp) X+d_{h, A, a}(\wp) \in O_{K}[X]$ by $R_{A, \wp, a}(X):=R_{A, \wp}(X+a)$.

Lemma 4.1. Let $A$ be an abelian variety over a number field $F$, as above. Let $\wp \in \mathcal{P}_{A}$ be a prime of ordinary reduction for $A$, and let $m$ be a positive integer such

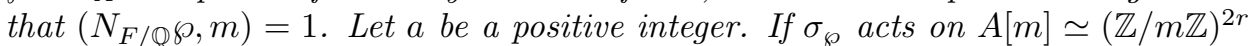
through the scalar matrix $a \cdot I_{2 r}$, then

$$
m^{k} \mid d_{k, A, a}(\wp),
$$

for any $k=1, \ldots, h$.

Proof. The proof is similar to the proof of Lemma 3.1.

Lemma 4.2. We have

$$
\left|\sigma_{i}\left(d_{k, A, a}(\wp)\right)\right| \leq(2+2 a)^{2 r} N_{F / \mathbb{Q}} \wp^{\frac{k}{2}},
$$

for any $i=1, \ldots, 2 e$ and for any $k=1, \ldots, h$. 
Proof. The proof is similar to the proof of Lemma 3.2.

Lemma 4.3. We have

$d_{h, A, a}(\wp)=d_{h, A}(\wp)+e_{1, a} d_{1, A, a}(\wp)+e_{2, a} d_{2, A, a}(\wp)+\ldots+e_{h-1, a} d_{h-1, A, a}(\wp)+e_{h, a}$, where $e_{1, a}, \ldots, e_{h, a}$ are integers which depend only on $h$ and $a$.

Proof. The proof is similar to the proof of Lemma 2.5.

Lemma 4.4. With the same notation as above, for any $m \in \mathbb{N}^{*}$ and any $x \in \mathbb{R}$, we have

$$
\begin{gathered}
\pi_{a}(x, F(A[m]) / F):=\mid\left\{\wp \in \mathcal{P}_{A} \mid N_{F / \mathbb{Q}} \wp \leq x,\left(m, N_{F / \mathbb{Q}} \wp\right)=1,\right. \\
\left.\sigma_{\wp} \text { acts on } A[m] \simeq(\mathbb{Z} / m \mathbb{Z})^{2 r} \text { through the scalar matrix } a \cdot I_{2 r}\right\} \mid \\
\ll \frac{x^{\frac{2 h^{2}-h+2}{2}}}{m^{2 h^{2}-h+2}}+1 .
\end{gathered}
$$

Proof. By using similar results as in Lemmas 3.1, 3.2, and 3.3 one can prove Lemma 4.4 in the same way as Lemma 3.4.

Lemma 4.5. Assume that $e=1$. Then, with the same notation as above, for any $m \in \mathbb{N}^{*}$ and any $x \in \mathbb{R}$, we have

$$
\begin{gathered}
\pi_{a}^{o}(x, F(A[m]) / F):=\mid\left\{\wp \in \mathcal{P}_{A} \mid N_{F / \mathbb{Q} \wp} \leq x,\left(m, N_{F / \mathbb{Q} \wp}\right)=1, \wp\right. \text { is ordinary, } \\
\text { and } \left.\sigma_{\wp} \text { acts on } A[m] \simeq(\mathbb{Z} / m \mathbb{Z})^{2 r} \text { through the scalar matrix } a \cdot I_{2 r}\right\} \mid \\
\ll \frac{x^{\frac{h^{2}+h}{2}}}{m^{h^{2}+h}}+1 .
\end{gathered}
$$

Proof. Since $e=1$, we have that $K=\mathbb{Q}(\sqrt{-D})$, where $D$ is a positive integer, and $O_{K} \subseteq \frac{1}{d}(\mathbb{Z}+\sqrt{-D} \mathbb{Z})$, for some positive integer $d$. Let $\wp \in \mathcal{P}_{A}$ be such that $N_{F / \mathbb{Q} \wp} \leq x,\left(m, N_{F / \mathbb{Q}} \wp\right)=1, \wp$ is ordinary, and $\sigma_{\wp}$ acts on $A[m] \simeq(\mathbb{Z} / m \mathbb{Z})^{2 r}$ through the scalar matrix $a \cdot I_{2 r}$. Then from Lemma 4.3 we have that $d_{h, A}(\wp)=$ $d_{h, A, a}(\wp)-e_{1, a} d_{1, A, a}(\wp)-e_{2, a} d_{2, A, a}(\wp)-\ldots-e_{h-1, a} d_{h-1, A, a}(\wp)-e_{h, a}$. But from Lemma 4.1 we know that $m^{k} \mid d_{k, A, a}(\wp)$, for any $k=1, \ldots, h$, and from Lemma 4.2 we know that $\left|d_{k, A, a}(\wp)\right| \leq(2+2 a)^{2 r} N_{F / \mathbb{Q}} \wp^{\frac{k}{2}}$, for any $k=1, \ldots, h$. Hence $d_{h, A}(\wp)=m^{h} d_{h}+\ldots+m d_{1}-e_{h, a}$, where $d_{1}, \ldots, d_{h} \in O_{K}$, such that $\left|d_{k}\right| \ll \frac{x^{\frac{k}{2}}}{m^{k}}$, for $k=1, \ldots, h$. By writing $d_{k}=e_{k}+\sqrt{-D} f_{k} \in O_{K} \subseteq \frac{1}{d}(\mathbb{Z}+\sqrt{-D} \mathbb{Z})$, where $e_{k}, f_{k} \in \frac{1}{d} \mathbb{Z}$, we get that

$$
\begin{aligned}
N_{F / \mathbb{Q} \wp^{h}} & =\operatorname{det} \rho_{l}\left(\sigma_{\wp}\right) \\
& =N_{K / \mathbb{Q}} d_{h, A}(\wp)=\left(m^{h} e_{h}+\ldots+m e_{1}-e_{h, a}\right)^{2}+D\left(m^{h} f_{h}+\ldots+m f_{1}\right)^{2},
\end{aligned}
$$

and also that $\left|e_{k}\right| \ll \frac{x^{\frac{k}{2}}}{m^{k}}$ and $\left|f_{k}\right| \ll \frac{x^{\frac{k}{2}}}{m^{k}}$, for $k=1, \ldots, h$. Hence

$$
\pi_{a}^{o}(x, F(A[m]) / F) \ll \prod_{k=1}^{h}\left(\frac{x^{\frac{k}{2}}}{m^{k}}+1\right)^{2} \ll \frac{x^{\frac{h^{2}+h}{2}}}{m^{h^{2}+h}}+1 .
$$




\section{Chebotarev Density theorem}

Let $L / F$ be a Galois extension of number fields, with Galois group $G$. We denote by $n_{L}$ and $d_{L}$ the degree and the discriminant of $L / \mathbb{Q}$, and by $d_{F}$ the discriminant of $F / \mathbb{Q}$. Let $\mathcal{P}(L / F)$ be the set of rational primes $p$ which lie below places of $F$ which ramify in $L / F$.

We know (see page 130 of $[\mathrm{SE}]$ ):

Lemma 5.1. If $L / F$ is a Galois extension of number fields, then

$$
\log d_{L} \leq|G| \log d_{F}+n_{L}\left(1-\frac{1}{|G|}\right) \sum_{p \in \mathcal{P}(L / F)} \log p+n_{L} \log |G| .
$$

Using the same assumptions as above, let $C$ be a conjugacy class in $G$. For a positive real number $x$, let

$$
\pi_{C}(x, L / F):=\mid\left\{\wp \in \Sigma_{F} \mid N_{F / \mathbb{Q}} \wp \leq x, \wp \text { unramified in } L / F, \sigma_{\wp} \in C\right\} \mid,
$$

where $\sigma_{\wp}$ is a Frobenius element at $\wp$. The Chebotarev density theorem says that

$$
\pi_{C}(x, L / F) \sim \frac{|C|}{|G|} \operatorname{li} x \sim \frac{|C|}{|G|} \frac{x}{\log x},
$$

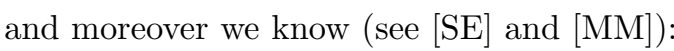

Lemma 5.2. Let $L / F$ be a Galois extension of number fields. If the Dedekind zeta function of $L$ satisfies the GRH, then

$$
\left|\pi_{C}(x, L / F)-\frac{|C|}{|G|} l i x\right| \ll|C| x^{\frac{1}{2}}\left(\log x+\frac{\log \left|d_{L}\right|}{|G|}\right),
$$

where the implied $O$-constant depends only on F. Moreover, if the Artin L-functions attached to the irreducible characters of G satisfy GRH, AHC, and PCC, then

$$
\left|\pi_{C}(x, L / F)-\frac{|C|}{|G|} l i x\right| \ll|C|^{\frac{1}{2}}\left(\frac{|\tilde{G}|}{|G|}\right)^{\frac{1}{4}} x^{\frac{1}{2}}\left(\log x\left(|G|\left(\prod_{p \in \mathcal{P}(L / F)} p\right)\right)\right),
$$

where $\tilde{G}$ denotes the set of conjugacy classes of $G$. The implied $O$-constant depends only on $F$.

\section{The Proof of Theorem 1.1}

We want to estimate the sum

$$
f_{A, a, F}(x):=\sum_{\substack{\wp \in \mathcal{P}_{A} \\ N_{F / \mathbb{Q} \wp \leq x}}}\left|\tau_{A, a, F}(\wp)\right| .
$$

If $m \in \tau_{A, a, F}(\wp)$, then $m^{2 r} \mid P_{A, \wp}(a)<(1+a)^{2 r} x^{r}$. Hence it is sufficient to consider only positive integers $m$ satisfying $m \leq(1+a) x^{\frac{1}{2}}$.

Thus

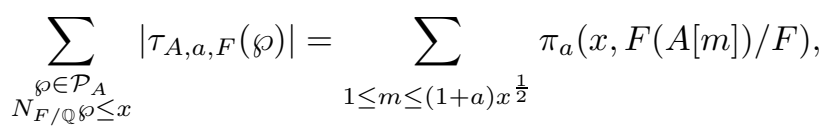

where

$$
\pi_{a}(x, F(A[m]) / F):=\mid\left\{\wp \in \mathcal{P}_{A} \mid N_{F / \mathbb{Q}} \wp \leq x,\left(m, N_{F / \mathbb{Q}} \wp\right)=1,\right.
$$

$\sigma_{\wp}$ acts on $A[m] \simeq(\mathbb{Z} / m \mathbb{Z})^{2 r}$ through the scalar matrix $\left.a \cdot I_{2 r}\right\} \mid$. 
If $y=y(x)$ is a real number with $y \leq(1+a) x^{\frac{1}{2}}$ ( $y$ will be chosen later), then

$$
\begin{gathered}
f_{A, a, F}(x)=\sum_{m \leq(1+a) x^{\frac{1}{2}}} \pi_{a}(x, F(A[m]) / F) \\
=\sum_{m \leq y} \pi_{a}(x, F(A[m]) / F)+\sum_{y<m \leq(1+a) x^{\frac{1}{2}}} \pi_{a}(x, F(A[m]) / F) \\
=\text { main }+ \text { error. }
\end{gathered}
$$

From Lemmas 5.2 and 2.1, under GRH, we get

$$
\begin{aligned}
\text { main } & =\sum_{m \leq y} \frac{1}{n(m)} \text { li } x+\sum_{m \leq y} O\left(x^{\frac{1}{2}} \log \left(m N_{F / \mathbb{Q}} \mathcal{N} x\right)\right) \\
& =\sum_{m \leq y} \frac{1}{n(m)} \operatorname{li} x+O\left(y x^{\frac{1}{2}} \log \left(N_{F / \mathbb{Q}} \mathcal{N} x\right)\right) .
\end{aligned}
$$

Now we estimate the error. For each $b=\left(b_{1}, \ldots, b_{2 r-1}\right) \in \mathbb{Z}^{2 r-1}$, with $\left|b_{k}\right| \leq$ $(2+2 a)^{2 r} x^{\frac{k}{2}}$ for $k=1, \ldots, 2 r-1$, and for each positive integer $m$ we define

$$
\begin{gathered}
S_{b, a}(m):=\left\{\wp \in \mathcal{P}_{A} \mid N_{F / \mathbb{Q}} \wp \leq x, b_{k, A, a}(\wp)=b_{k} \text { for } k=1, \ldots, 2 r-1,\right. \\
\left.\sigma_{\wp} \text { acts on } A[m] \simeq(\mathbb{Z} / m \mathbb{Z})^{2 r} \text { through the scalar matrix } a \cdot I_{2 r}\right\} .
\end{gathered}
$$

Then, because from Lemma 2.4 we know that $\left|b_{k, A, a}(\wp)\right| \leq(2+2 a)^{2 r} x^{\frac{k}{2}}$, for $k=1, \ldots, 2 r$, we obtain

$$
\text { error } \leq \sum_{y<m \leq(1+a) x^{\frac{1}{2}}} \sum_{\substack{b \in \mathbb{Z}^{2 r-1} \\\left|b_{k}\right| \leq(2+2 a)^{2 r} x^{\frac{k}{2}}, \text { for } k=1, \ldots, 2 r-1}}\left|S_{b, a}(m)\right| .
$$

From Lemma 2.3 we know that for each $\wp \in S_{b, a}(m)$ we have $m^{k} \mid b_{k, A, a}(\wp)$, for $k=$ $1, \ldots, 2 r$, and from Lemma 2.5 we know that $b_{2 r, A, a}(\wp)=N_{F / \mathbb{Q}} \wp^{r}+c_{1} b_{1, A, a}(\wp)+$ $c_{2} b_{2, A, a}(\wp)+\ldots+c_{2 r-1} b_{2 r-1, A, a}(\wp)+c_{2 r}$, and therefore

$$
\begin{aligned}
& \sum_{y<m \leq(1+a) x^{\frac{1}{2}}} \sum_{\substack{b \in \mathbb{Z}^{2 r-1} \\
\left|b_{k}\right| \leq(2+2 a)^{2 r}}}\left|S_{b, a}(m)\right| \\
& \leq \sum_{y<m \leq(1+a) x^{\frac{1}{2}}} \sum_{\substack{b \in \mathbb{Z}^{2 r-1} \\
\left|b_{k}\right| \leq(2+2 a)^{2 r} x^{\frac{k}{2}}, \text { for } k=1, \ldots, 2 r-1 \\
m^{k} \mid b_{k}, \text { for } k=1, \ldots, 2 r-1}} \\
& \sum_{\wp \in \mathcal{P}_{A}} \\
& N_{F / \mathbb{Q} \wp \leq x} \\
& b_{k, A}(\wp)=b_{k} \text {, for } k=1, \ldots, 2 r-1 \\
& m^{2 r} \mid b_{2 r, A}(\wp)=N_{F / \mathbb{Q}} \wp^{r}+c_{1} b_{1} \\
& \ll \sum_{y<m \leq(1+a) x^{\frac{1}{2}}} \sum_{\substack{b \in \mathbb{Z}^{2 r-1} \\
\left|b_{k}\right| \leq(2+2 a)^{2 r} x^{\frac{k}{2}}, \text { for } k=1, \ldots, 2 r-1 \\
m^{k} \mid b_{k}, \text { for } k=1, \ldots, 2 r-1}}\left(\frac{x}{m^{2}}+1\right) \\
& \ll \sum_{y<m \leq(1+a) x^{\frac{1}{2}}}\left(\frac{x}{m^{2}}+1\right) \prod_{k=1}^{2 r-1}\left(\frac{x^{\frac{k}{2}}}{m^{k}}+1\right) \\
& \ll \sum_{y<m \leq(1+a) x^{\frac{1}{2}}}\left(\frac{x}{m^{2}}+1\right)\left(\frac{x^{\frac{2 r^{2}-r}{2}}}{m^{2 r^{2}-r}}+1\right) \ll \frac{x^{\frac{2 r^{2}-r+2}{2}}}{y^{2 r^{2}-r+1}} .
\end{aligned}
$$


From the above we get

$$
f_{A, a, F}(x)=\sum_{m \leq y} \frac{1}{n(m)} \operatorname{li} x+O\left(y x^{\frac{1}{2}} \log x\right)+O\left(\frac{x^{\frac{2 r^{2}-r+2}{2}}}{y^{2 r^{2}-r+1}}\right) .
$$

We choose $y$ such that $x^{\frac{1}{2}} y \log x=\frac{\frac{2 r^{2}-r+2}{2}}{y^{2 r^{2}-r+1}}$, i.e.

$$
y:=\frac{x^{\frac{2 r^{2}-r+1}{4 r^{2}-2 r+4}}}{(\log x)^{\frac{1}{2 r^{2}-r+2}}} .
$$

Then

$$
f_{A, a, F}(x)=\sum_{m \leq y} \frac{1}{n(m)} \operatorname{li} x+O\left(x^{\frac{4 r^{2}-2 r+3}{4 r^{2}-2 r+4}}(\log x)^{\frac{2 r^{2}-r+1}{2 r^{2}-r+2}}\right) .
$$

From Lemma 2.2, with $\epsilon=\frac{1}{2}$, we obtain

$$
\sum_{m>y} \frac{1}{n(m)} \ll \sum_{m>y} \frac{1}{m^{\frac{3}{2}}} \ll \frac{1}{y^{\frac{1}{2}}} .
$$

Since $y:=\frac{x^{\frac{2 r^{2}-r+1}{4 r^{2}-2 r+4}}}{(\log x)^{\frac{1}{2 r^{2}-r+2}}}$, we get

$$
f_{A, a, F}(x)=c_{A, F} \operatorname{li} x+O\left(x^{\frac{4 r^{2}-2 r+3}{4 r^{2}-2 r+4}}(\log x)^{\frac{2 r^{2}-r+1}{2 r^{2}-r+2}}\right),
$$

and Theorem 1.1 is proved.

\section{The proof of Theorem 1.2}

As in $\S 6$ above we have

$$
f_{A, a, F}(x)=\text { main }+ \text { error }
$$

and

$$
\text { main }=\sum_{m \leq y} \frac{1}{n(m)} \operatorname{li} x+O\left(y x^{\frac{1}{2}} \log \left(N_{F / \mathbb{Q}} \mathcal{N} x\right)\right) .
$$

From Lemma 3.4 we know that

$$
\begin{gathered}
\text { error } \leq \sum_{y<m \leq(1+a) x^{\frac{1}{2}}} \pi_{a}(x, F(A[m]) / F) \ll \sum_{y<m \leq(1+a) x^{\frac{1}{2}}}\left(\frac{x^{\frac{2 h^{2}-h+2}{2}}}{m^{2 h^{2}-h+2}}+1\right) \\
\ll \frac{x^{\frac{2 h^{2}-h+2}{2}}}{y^{2 h^{2}-h+1}} .
\end{gathered}
$$

Hence

$$
f_{A, a, F}(x)=\sum_{m \leq y} \frac{1}{n(m)} \operatorname{li} x+O\left(y x^{\frac{1}{2}} \log x\right)+O\left(\frac{x^{\frac{2 h^{2}-h+2}{2}}}{y^{2 h^{2}-h+1}}\right) .
$$

We choose $y$ such that $x^{\frac{1}{2}} y \log x=\frac{x^{\frac{2 h^{2}-h+2}{2}}}{y^{2 h^{2}-h+1}}$, i.e.

$$
y:=\frac{x^{\frac{2 h^{2}-h+1}{4 h^{2}-2 h+4}}}{(\log x)^{\frac{1}{2 h^{2}-h+2}}} .
$$


Then

$$
f_{A, a, F}(x)=\sum_{m \leq y} \frac{1}{n(m)} \text { li } x+O\left(x^{\frac{4 h^{2}-2 h+3}{4 h^{2}-2 h+4}}(\log x)^{\frac{2 h^{2}-h+1}{2 h^{2}-h+2}}\right) .
$$

From Lemma 2.2, with $\epsilon=\frac{1}{2}$, we obtain

$$
\sum_{m>y} \frac{1}{n(m)} \ll \sum_{m>y} \frac{1}{m^{\frac{3}{2}}} \ll \frac{1}{y^{\frac{1}{2}}} .
$$

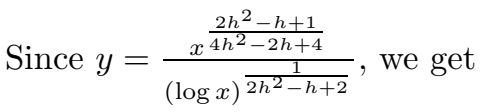

$$
f_{A, a, F}(x)=c_{A, F} \operatorname{li} x+O\left(x^{\frac{4 h^{2}-2 h+3}{4 h^{2}-2 h+4}}(\log x)^{\frac{2 h^{2}-h+1}{2 h^{2}-h+2}}\right),
$$

and Theorem 1.2 is proved.

\section{The Proof of TheOrem 1.3}

By using Lemma 4.4 one can prove the first part of Theorem 1.3 in the same way as Theorem 1.2.

Now we prove the second part of Theorem 1.3. As in $\S 7$ above we have

$$
f_{A, a, F}(x)=\text { main }+ \text { error }
$$

and

$$
\text { main }=\sum_{m \leq y} \frac{1}{n(m)} \operatorname{li} x+O\left(y x^{\frac{1}{2}} \log \left(N_{F / \mathbb{Q}} \mathcal{N} x\right)\right) .
$$

Now we estimate the error. We have

$$
\text { error } \leq \sum_{y<m \leq(1+a) x^{\frac{1}{2}}} \pi_{a}^{o}(x, F(A[m]) / F)+\sum_{y<m \leq(1+a) x^{\frac{1}{2}}} \pi_{a}^{s}(x, F(A[m]) / F),
$$

where

$$
\pi_{a}^{o}(x, F(A[m]) / F):=\mid\left\{\wp \in \mathcal{P}_{A} \mid N_{F / \mathbb{Q}} \wp \leq x, \wp\right. \text { has ordinary reduction and }
$$$$
\left.\sigma_{\wp} \text { acts on } A[m] \simeq(\mathbb{Z} / m \mathbb{Z})^{2 r} \text { through the scalar matrix } a \cdot I_{2 r}\right\} \mid
$$

and

$$
\begin{gathered}
\pi_{a}^{s}(x, F(A[m]) / F):=\mid\left\{\wp \in \mathcal{P}_{A} \mid N_{F / \mathbb{Q}} \wp \leq x, \wp\right. \text { has supersingular reduction and } \\
\left.\sigma_{\wp} \text { acts on } A[m] \simeq(\mathbb{Z} / m \mathbb{Z})^{2 r} \text { through the scalar matrix } a \cdot I_{2 r}\right\} \mid .
\end{gathered}
$$

From Lemma 4.5 we get that

$$
\begin{gathered}
\sum_{y<m \leq(1+a) x^{\frac{1}{2}}} \pi_{a}^{o}(x, F(A[m]) / F) \ll \sum_{y<m \leq(1+a) x^{\frac{1}{2}}}\left(\frac{x^{\frac{h^{2}+h}{2}}}{m^{h^{2}+h}}+1\right) \\
\ll \frac{x^{\frac{h^{2}+h}{2}}}{y^{h^{2}+h-1}} .
\end{gathered}
$$

If $\sigma_{\wp}$ acts on $A[m] \simeq(\mathbb{Z} / m \mathbb{Z})^{2 r}$ through the scalar matrix $a \cdot I_{2 r}$, then $m^{2 r} \mid P_{A, \wp}(a)$ $=\left(N_{F / \mathbb{Q}} \wp+a^{2}\right)^{r}$ (see Lemma 2.3) and also $m \mid N_{F / \mathbb{Q}} \wp-a^{2}$ (see item 2 of Lemma 2.1 above), and hence $m \mid 2 a^{2}$. Thus we choose $y>2 a^{2}$, and we get

$$
\sum_{y<m \leq(1+a) x^{\frac{1}{2}}} \pi_{a}^{s}(x, F(A[m]) / F)=0 .
$$


From the above we obtain

$$
f_{A, a, F}(x)=\sum_{m \leq y} \frac{1}{n(m)} \operatorname{li} x+O\left(y x^{\frac{1}{2}} \log x\right)+O\left(\frac{x^{\frac{h^{2}+h}{2}}}{y^{h^{2}+h-1}}\right) .
$$

We choose $y$ such that $x^{\frac{1}{2}} y \log x=\frac{x^{\frac{h^{2}+h}{2}}}{y^{h^{2}+h-1}}$, i.e.

$$
y:=\frac{x^{\frac{h^{2}+h-1}{2 h^{2}+2 h}}}{(\log x)^{\frac{1}{h^{2}+h}}} .
$$

Then

$$
f_{A, a, F}(x)=\sum_{m \leq y} \frac{1}{n(m)} \operatorname{li} x+O\left(x^{\frac{2 h^{2}+2 h-1}{2 h^{2}+2 h}}(\log x)^{\frac{h^{2}+h-1}{h^{2}+h}}\right) .
$$

From Lemma 2.2, with $\epsilon=\frac{1}{2}$, we obtain

$$
\sum_{m>y} \frac{1}{n(m)} \ll \sum_{m>y} \frac{1}{m^{\frac{3}{2}}} \ll \frac{1}{y^{\frac{1}{2}}} .
$$

Since $y=\frac{x^{\frac{h^{2}+h-1}{2 h^{2}+2 h}}}{(\log x)^{\frac{1}{h^{2}+h}}}$, we get

$$
f_{A, a, F}(x)=c_{A, F} \operatorname{li} x+O\left(x^{\frac{2 h^{2}+2 h-1}{2 h^{2}+2 h}}(\log x)^{\frac{h^{2}+h-1}{h^{2}+h}}\right),
$$

and Theorem 1.3 is proved.

\section{The Proof of TheOrem 1.4}

As in $\S 7$ above we have

$$
f_{A, a, F}(x)=\text { main }+ \text { error }
$$

and

$$
\text { error }=O\left(\frac{x^{\frac{2 r^{2}-r+2}{2}}}{y^{2 r^{2}-r+1}}\right) \text {. }
$$

Now we estimate main. Fix $\epsilon>0$. Then from Lemmas 5.2, 5.1, 2.1, and Corollary 3.6, under GRH, AHC, and PCC, we get

$$
\begin{gathered}
\text { main }=\sum_{m \leq y} \frac{1}{n(m)} \operatorname{li} x+\sum_{m \leq y} O\left(\left(\frac{\left|\tilde{G}_{m}\right|}{\left|G_{m}\right|}\right)^{\frac{1}{4}} x^{\frac{1}{2}} \log \left(m N_{F / \mathbb{Q}} \mathcal{N} x\right)\right) \\
\ll \sum_{m \leq y} \frac{1}{n(m)} \operatorname{li} x+\sum_{m \leq y} O\left(\left(m^{-e\left(2 h^{2}-h+1\right)+\epsilon}\right)^{\frac{1}{4}} x^{\frac{1}{2}} \log \left(m N_{F / \mathbb{Q}} \mathcal{N} x\right)\right) \\
=\sum_{m \leq y} \frac{1}{n(m)} \operatorname{li} x+O\left(y^{1-\frac{2 e h^{2}-e h+e-\epsilon}{4}} x^{\frac{1}{2}} \log \left(N_{F / \mathbb{Q}} \mathcal{N} x\right)\right) .
\end{gathered}
$$

From the above we get

$$
f_{A, a, F}(x)=\sum_{m \leq y} \frac{1}{n(m)} \operatorname{li} x+O\left(y^{1-\frac{2 e h^{2}-e h+e-\epsilon}{4}} x^{\frac{1}{2}} \log x\right)+O\left(\frac{x^{\frac{2 r^{2}-r+2}{2}}}{y^{2 r^{2}-r+1}}\right) .
$$


We choose $y$ such that $x^{\frac{1}{2}} y^{1-\frac{2 e h^{2}-e h+e-\epsilon}{4}} \log x=\frac{x^{\frac{2 r^{2}-r+2}{2}}}{y^{2 r^{2}-r+1}}$, i.e.

$$
y:=\frac{x^{\frac{4 r^{2}-2 r+2}{8 r^{2}-4 r+8-2 e h^{2}+e h-e+\epsilon}}}{(\log x)^{\overline{8 r^{2}-4 r+8-2 e h^{2}+e h-e+\epsilon}}} .
$$

Then

$$
\begin{gathered}
f_{A, a, F}(x)=\sum_{m \leq y} \frac{1}{n(m)} \operatorname{li} x \\
+O\left(x^{\frac{2 r^{2}-r+2}{2}-\left(2 r^{2}-r+1\right) \frac{4 r^{2}-2 r+2}{8 r^{2}-4 r+8-2 e h^{2}+e h-e+\epsilon}}(\log x)^{\frac{8 r^{2}-4 r+4}{8 r^{2}-4 r+8-2 e h^{2}+e h-e+\epsilon}}\right) .
\end{gathered}
$$

From Lemma 3.5, we obtain

$$
\begin{gathered}
\sum_{m>y} \frac{1}{n(m)} \ll \sum_{m>y} \frac{1}{m^{2 h^{2} e+h e+1-\epsilon}} \\
\ll \frac{1}{y^{2 h^{2} e+h e-\epsilon}}=\frac{x^{-\left(2 h^{2} e+h e-\epsilon\right)\left(\frac{4 r^{2}-2 r+2}{8 r^{2}-4 r+8-2 e h^{2}+e h-e+\epsilon}\right)}}{(\log x)^{\frac{8 h^{2} e+4 h e-4 \epsilon}{8 r^{2}-4 r+8-2 e h^{2}+e h-e+\epsilon}}} .
\end{gathered}
$$

Hence, for $\epsilon>0$ sufficiently small, we get

$$
\begin{gathered}
f_{A, a, F}(x)=c_{A, F} \operatorname{li} x \\
+O\left(x^{\frac{2 r^{2}-r+2}{2}-\left(2 r^{2}-r+1\right) \frac{4 r^{2}-2 r+2}{8 r^{2}-4 r+8-2 e h^{2}+e h-e+\epsilon}}(\log x)^{\frac{8 r^{2}-4 r+4}{8 r^{2}-4 r+8-2 e h^{2}+e h-e+\epsilon}}\right),
\end{gathered}
$$

and Theorem 1.4 is proved (because we can choose $\epsilon>0$ arbitrarily small).

\section{The PRoOF OF TheOREM 1.5}

As in $\S 7$ above we have

$$
f_{A, a, F}(x)=\text { main }+ \text { error }
$$

and

$$
\text { error }=O\left(\frac{x^{\frac{2 r^{2}-r+2}{2}}}{y^{2 r^{2}-r+1}}\right) \text {. }
$$

Now we estimate main. Fix $\epsilon>0$. Then from Lemmas 5.2, 5.1, 2.1, and Corollary 3.8, under GRH, AHC, and PCC, we get

$$
\begin{gathered}
\text { main }=\sum_{m \leq y} \frac{1}{n(m)} \operatorname{li} x+\sum_{m \leq y} O\left(\left(\frac{\left|\tilde{G}_{m}\right|}{\left|G_{m}\right|}\right)^{\frac{1}{4}} x^{\frac{1}{2}} \log \left(m N_{F / \mathbb{Q}} \mathcal{N} x\right)\right) \\
\ll \sum_{m \leq y} \frac{1}{n(m)} \operatorname{li} x+\sum_{m \leq y} O\left(\left(m^{-e h^{2}+e h+\epsilon}\right)^{\frac{1}{4}} x^{\frac{1}{2}} \log \left(m N_{F / \mathbb{Q}} \mathcal{N} x\right)\right) \\
=\sum_{m \leq y} \frac{1}{n(m)} \operatorname{li} x+O\left(y^{1-\frac{e h^{2}-e h-\epsilon}{4}} x^{\frac{1}{2}} \log \left(N_{F / \mathbb{Q}} \mathcal{N} x\right)\right) .
\end{gathered}
$$

From the above we get

$$
f_{A, a, F}(x)=\sum_{m \leq y} \frac{1}{n(m)} \operatorname{li} x+O\left(y^{1-\frac{e h^{2}-e h-\epsilon}{4}} x^{\frac{1}{2}} \log x\right)+O\left(\frac{x^{\frac{2 r^{2}-r+2}{2}}}{y^{2 r^{2}-r+1}}\right) .
$$


We choose $y$ such that $x^{\frac{1}{2}} y^{1-\frac{e h^{2}-e h-\epsilon}{4}} \log x=\frac{x^{\frac{2 r^{2}-r+2}{2}}}{y^{2 r^{2}-r+1}}$, i.e.

$$
y:=\frac{x^{\frac{4 r^{2}-2 r+2}{8 r^{2}-4 r+8-e h^{2}+e h+\epsilon}}}{(\log x)^{\overline{8 r^{2}-4 r+8-e h^{2}+e h+\epsilon}}} .
$$

Then

$$
\begin{gathered}
f_{A, a, F}(x)=\sum_{m \leq y} \frac{1}{n(m)} \operatorname{li} x \\
+O\left(x^{\frac{2 r^{2}-r+2}{2}-\left(2 r^{2}-r+1\right) \frac{4 r^{2}-2 r+2}{8 r^{2}-4 r+8-e h^{2}+e h+\epsilon}}(\log x)^{\frac{8 r^{2}-4 r+4}{8 r^{2}-4 r+8-e h^{2}+e h+\epsilon}}\right) .
\end{gathered}
$$

From Lemma 3.5, we obtain

$$
\begin{gathered}
\sum_{m>y} \frac{1}{n(m)} \ll \sum_{m>y} \frac{1}{m^{h^{2} e+1-\epsilon}} \\
\ll \frac{1}{y^{h^{2} e-\epsilon}}=\frac{\left.x^{-\left(h^{2} e-\epsilon\right)\left(\frac{4 r^{2}-2 r+2}{8 r^{2}-4 r+8-e h^{2}+e h+\epsilon}\right.}\right)}{(\log x)^{\frac{4 h^{2} e-4 \epsilon}{8 r^{2}-4 r+8-e h^{2}+e h+\epsilon}} .}
\end{gathered}
$$

Hence, for $\epsilon>0$ sufficiently small, we get

$$
\begin{gathered}
f_{A, a, F}(x)=c_{A, F} \operatorname{li} x \\
+O\left(x^{\frac{2 r^{2}-r+2}{2}-\left(2 r^{2}-r+1\right) \frac{4 r^{2}-2 r+2}{8 r^{2}-4 r+8-e h^{2}+e h+\epsilon}}(\log x)^{\frac{8 r^{2}-4 r+4}{8 r^{2}-4 r+8-e h^{2}+e h+\epsilon}}\right),
\end{gathered}
$$

and Theorem 1.5 is proved (because we can choose $\epsilon>0$ arbitrarily small).

\section{RefEREnCES}

[AG] Amir Akbary and Dragos Ghioca, A geometric variant of Titchmarsh divisor problem, Int. J. Number Theory 8 (2012), no. 1, 53-69, DOI 10.1142/S1793042112500030. MR2887882

[BGK1] G. Banaszak, W. Gajda, and P. Krason, On the image of l-adic Galois representations for abelian varieties of type I and II, Doc. Math. Extra Vol. (2006), 35-75 (electronic). MR2290584(2008i:11071)

[BGK2] Grzegorz Banaszak, Wojciech Gajda, and Piotr Krason, On the image of Galois l-adic representations for abelian varieties of type III, Tohoku Math. J. (2) 62 (2010), no. 2, 163-189, DOI 10.2748/tmj/1277298644. MR2663452(2011h:14054)

[CM] Alina Carmen Cojocaru and M. Ram Murty, Cyclicity of elliptic curves modulo $p$ and elliptic curve analogues of Linnik's problem, Math. Ann. 330 (2004), no. 3, 601-625, DOI 10.1007/s00208-004-0562-x. MR2099195 (2005k:11108)

[M] David Mumford, Abelian varieties, Tata Institute of Fundamental Research Studies in Mathematics, vol. 5, Published for the Tata Institute of Fundamental Research, Bombay; by Hindustan Book Agency, New Delhi, 2008. With appendices by C. P. Ramanujam and Yuri Manin; Corrected reprint of the second (1974) edition. MR2514037 (2010e:14040)

[SE] Jean-Pierre Serre, Quelques applications du théorème de densité de Chebotarev (French), Inst. Hautes Études Sci. Publ. Math. 54 (1981), 323-401. MR644559 (83k:12011)

[SE1] Jean-Pierre Serre, Un critère d'indépendance pour une famille de représentations $\ell-$ adiques (French, with English summary), Comment. Math. Helv. 88 (2013), no. 3, 541554, DOI 10.4171/CMH/295. MR3093502 
[SH] Goro Shimura, Introduction to the arithmetic theory of automorphic functions, Publications of the Mathematical Society of Japan, No. 11. Iwanami Shoten, Publishers, Tokyo; Princeton University Press, Princeton, N.J., 1971. Kanô Memorial Lectures, No. 1. MR0314766 (47 \#3318)

[SI] Joseph H. Silverman, Advanced topics in the arithmetic of elliptic curves, Graduate Texts in Mathematics, vol. 151, Springer-Verlag, New York, 1994. MR1312368 (96b:11074)

Department of Mathematics, Yonsei University, Seoul 120-749, Republic of Korea

E-mail address: cristian.virdol@gmail.com 